Systematic Review

\title{
RSA migration of unicondylar knee arthroplasties is comparable to migration of total knee arthroplasties: A meta-analysis
}

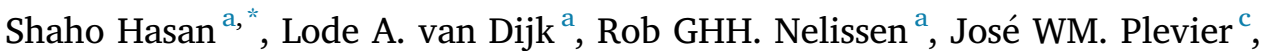 \\ Leendert Blankevoort ${ }^{\mathrm{b}}$, Bart G. Pijls ${ }^{\mathrm{a}}$ \\ ${ }^{a}$ Department of Orthopaedics, Leiden University Medical Centre, Leiden, the Netherlands \\ ${ }^{\mathrm{b}}$ Amsterdam UMC, University of Amsterdam, Department of Orthopaedics, Amsterdam Movement Sciences, Amsterdam, the Netherlands \\ ${ }^{\mathrm{c}}$ Walaeus Library, Leiden University Medical Centre, Leiden, the Netherlands
}

\section{A R T I C L E I N F O}

\section{Keywords:}

Knee $<$ anatomic location

Arthroplasty

Replacement

\begin{abstract}
A B S T R A C T
Importance: Aseptic loosening is a major cause of failure for unicondylar knee arthroplasty (UKA). In total knee arthroplasty (TKA), early migration as measured with radiostereometric analysis (RSA) is a strong predictor of late revision for aseptic loosening of the tibial component. Migration in the first two years provides information on the fixation of an implant. However, the migration pattern of UKAs has not been systematically determined, and it is unclear if the migration pattern of UKAs is similar to that of TKAs. Therefore, the present meta-analysis aims to evaluate the migration patterns of tibial components of UKAs.

Evidence review: All RSA studies reporting on migration at two or more postoperative time-points following UKA were included. Pubmed, Web of Science, Cochrane, and Embase were searched up to April 2021. The risk of bias was assessed using the methodological score of the Assessment of Quality in Lower Limb Arthroplasty tool. All phases of the review were performed by two reviewers independently. A random-effects model was applied to pool the migration data.

Findings: The literature search yielded 3,187 hits, of which ten studies were included, comprising 13 study groups and 381 UKAs. The majority of the early migration occurred in the first 6 months postoperatively followed by a period of very little migration, similar to what is reported for TKAs. The pooled mean migration expressed as the maximum total point motion of all UKAs at 3 months, 6 months, 1 year, and 2 years was $0.43 \mathrm{~mm}$ (95\% CI 0.38-0.48), $0.54 \mathrm{~mm}$ (95\% CI 0.40-0.67), 0.59 mm (95\% CI 0.52-0.66), and $0.61 \mathrm{~mm}$ (95\% CI 0.55-0.68), respectively. Migration at one year and two years was higher than migration of TKAs as reported in previous studies. All-polyethylene UKAs migrated more at one year (0.69 mm; 95\% CI 0.58-0.80) than metal-backed UKAs (0.52 mm; 95\% CI 0.46-0.58).

Conclusions and relevance: The migration pattern of UKAs is comparable with that of TKAs in the first two years as both types of implants show initial migration in the first few months and very little migration thereafter. However, UKAs had higher migration at 1-year and 2-year follow-up.
\end{abstract}

\section{Introduction}

Unicondylar knee arthroplasty (UKA) has the potential to treat medial and lateral knee osteoarthritis without replacement of the entire knee joint [1]. Although the popularity of UKA is increasing, isolated medial or lateral osteoarthritis of the knee is mostly treated with total knee arthroplasty (TKA). This difference is reflected in arthroplasty registries, with 90,147 TKA and 11,916 UKA surgeries having been registered in the National Joint Registry (NJR; England, Wales, Northern Ireland) in 2019 [2]. UKA has several advantages over TKA, such as

\footnotetext{
* Corresponding author. Albinusdreef 2, Leiden, 2333ZA, the Netherlands.

E-mail address: S.Hasan@lumc.nl (S. Hasan).
}

shorter operation time, shorter length of stay, decreased risk of early complications (e.g., deep infection, myocardial infarction), greater range of motion, and higher patient-reported outcome scores [3]. However, one of the major disadvantages is a higher mean revision rate of UKAs, with reported rates of $8.3 \%-11.0 \%$ for UKA as compared with $3.4 \%$ $4.2 \%$ for TKA [2]. The main reason for revision of a UKA is aseptic loosening, followed by dislocation or subluxation and pain [2].

In TKA, early migration (i.e., 1 to 2 years) has been associated with late revision (i.e., 5 to 10 years) for aseptic loosening [4]. Additionally, continuous migration after the first post-operative year has been associated with an early onset of aseptic loosening [5]. Moreover, three thresholds for migration at 1 year have been proposed to assess the risk of

https://doi.org/10.1016/j.jisako.2021.12.002

Available online 24 December 2021

2059-7754/@ 2021 The Author(s). Published by Elsevier Inc. on behalf of International Society of Arthroscopy, Knee Surgery and Orthopedic Sports Medicine. This is an open access article under the CC BY license (http://creativecommons.org/licenses/by/4.0/). 


\section{What is already known?}

- Migration profiles of TKA include high initial migration in the first few months and stabilization thereafter

- TKA migrating $>0.2 \mathrm{~mm}$ maximum total point motion (MTPM) between year 1 and year 2 are at risk for failure due to aseptic loosening

- Three other thresholds for migration at 1 year have been proposed to assess the risk of tibial loosening of total knee arthroplasty (TKA): $<0.54 \mathrm{~mm}$ MTPM, 0.54-1.6 mm MTPM, and $>1.6 \mathrm{~mm}$ MTPM

- No migration profiles or thresholds for unicondylar knee arthroplasty (UKA) are reported

tibial loosening of TKA and to classify TKAs as acceptable $(<0.54 \mathrm{~mm}$ maximum total point motion [MTPM]), at-risk (0.54-1.6 mm MTPM), or unacceptable ( $>1.6 \mathrm{~mm}$ MTPM) [4]. Therefore, the migration pattern provides important information on implant safety. The migration pattern can be measured very accurately with radiostereometric analysis (RSA), which has an accuracy of $0.2 \mathrm{~mm}$ [5]. While the migration pattern has been established for TKA, it is unknown for UKA, and it is unclear how the UKA migration pattern compares to the TKA migration pattern. Therefore, the present meta-analysis aimed to evaluate the migration patterns of tibial components of UKAs. The question was whether the postoperative migration pattern and magnitudes of migration of UKAs were the same as those reported for TKAs. The hypothesis that UKAs had a comparable migration pattern, as well as comparable magnitude of migration up to two years as TKAs was tested.

\section{Methods}

This meta-analysis was performed in concordance with the PRISMA statement [6]. The systematic review comprised migration patterns from RSA studies. The methodology of the review is the same as previously described for TKAs [7]. The present review was not registered.

\section{Literature search}

The literature search was conducted by a medical librarian (JP). RSA studies were searched up to October 2019 and were updated for studies up to April 2021 using Pubmed, Web of Science, Cochrane, and Embase. The search included a combination of the terms defining "RSA" and "Joint Replacement" (Appendix A). We decided to conduct a broad search, including all joint replacements, instead of focussing on UKA, in order to minimise the possibility of missing studies. Studies in English, Dutch, German, French, Spanish, and Italian were considered.

\section{Inclusion and exclusion analysis}

All RSA studies reporting migration patterns following UKA were identified. A migration pattern was defined as the reporting of migration at two or more postoperative time-points within the first 2 years of follow-up using the maximum total point motion (MTPM) [7]. The MTPM is defined as the point on the implant with the highest migration relative to the bone and is the most frequently reported outcome measure in RSA studies to report migration [4,5]. Titles and abstracts were screened by two reviewers (SH, LD) independently. If the reviewers disagreed, the study remained eligible, and the full text was screened. The eligible full texts were screened by the same two reviewers independently, and any disagreements were resolved by discussion or after consulting a third reviewer (BP). Inclusion criteria for the RSA studies were: (1) primary UKA and (2) MTPM measured with RSA. Studies with fewer than five UKAs and non-clinical studies (e.g., phantom or animal studies) were excluded. If the same cohort was reported in multiple

\section{What are the new findings?}

- The migration pattern of UKA was comparable with that of TKA, with high initial migration followed by a stabilization phase between 1-year and 2-year follow-up

- Migration of the UKA tibial components was higher at 1-year and 2-year follow-up compared to TKA

- Future studies should assess whether TKA thresholds are applicable for UKAs

publications, the publication with the longest follow-up was formally included, while the other publications were used for additional data if required.

\section{Data extraction}

Two investigators ( $\mathrm{SH}$ and $\mathrm{LD}$ ) independently extracted the data using a predefined SPSS database (IBM SPSS Statistics 26.0; IBM Corp, Armonk, NY, USA). Data extracted were first author, journal, year of publication, implant design, fixation method (i.e., cemented, uncemented), anatomical compartment (i.e., medial, lateral, both), insert (i.e., fixed, mobile), material (i.e., metal-backed, all-polyethylene), follow-up in years, and RSA technique (i.e., marker-based, model-based). Marker-based RSA is a technique that relies on movements between markers attached to or inserted into the prosthesis and markers positioned in the surrounding bone. In contrast, model-based RSA measures migration by comparing movements between a prosthesis model and markers positioned in the surrounding bone. Although these techniques have obvious differences, calculated MTPM from these different techniques do not show significant differences and can be pooled [8]. The number of patients at baseline and during follow-up was extracted, as was age, sex, and RSA results (i.e., MTPM values) 3 months, 4 months, 6 months, 1 year, and 2 years. For the purpose of pooling data, MTPM values at 3 and 4 months were combined as a single time point. The standard deviation or standard error was extracted. Some studies reported the MTPM graphically. In such cases, the MTPM was measured in the graphs by both reviewers, and the average of both measurements was taken.

\section{Quality assessment}

The risk of bias was assessed using the methodological score of the Assessment of Quality in Lower Limb Arthroplasty (AQUILA) tool [4,9]. The AQUILA tool was designed to assess the quality of observational studies in lower limb arthroplasty. Two investigators (SH and LD) assessed the risk of bias individually, and any discrepancy was resolved by discussion.

\section{Data analysis}

Migration patterns of included UKAs were plotted for up to 2 years. A pooled mean was calculated using a random-effects model, weighting means according to their standard error (se)[10]. If these was missing, it was calculated by subtracting the lower limit of the $95 \%$ confidence interval $(95 \% \mathrm{CI})$ from the upper limit of the and by dividing this difference by $3.92(2 * 1.96)$. If the standard deviation was missing, it was calculated by dividing the standard error by the square root of the number of included patients [11]. To assess the migration pattern of UKAs, the MTPMs of UKAs were pooled at each time point (i.e., 3-4 months, 6 months, 1 year, 2 years). Secondary migration of metal-backed tibial (MBT) and all-polyethylene tibial (APT) components was separately analysed and compared. In a post-hoc analysis, mean migration at 1 year was plotted against publication year in order to assess the influence of 
time on the migration of UKAs. The Metafor Package in R Statistics (version 3.6.1; R Foundation for Statistical Computing, Vienna, Austria) was used for the analysis [10].

Results

Inclusion of RSA studies

A total of 3,187 records were found in the initial search, of which 1,679 were duplicates, leaving 1,508 records to be screened based on title and abstract. Another 1,476 records were excluded for not involving an UKA $(\mathrm{k}=1,465)$, not being an RSA study $(\mathrm{k}=7)$, not being a clinical study $(\mathrm{k}=3)$ and one full-text could not be found $(\mathrm{k}=1)$. The full texts of 32 records were screened for eligibility, and 22 records were excluded (including 13 records that did not report the MTPM, 5 records that did not report a migration pattern, 2 records that used the same cohort, and 2 records that included less than 5 UKAs), leaving ten records to be included (Fig. 1, Table 1). Risk of bias of included studies is included in
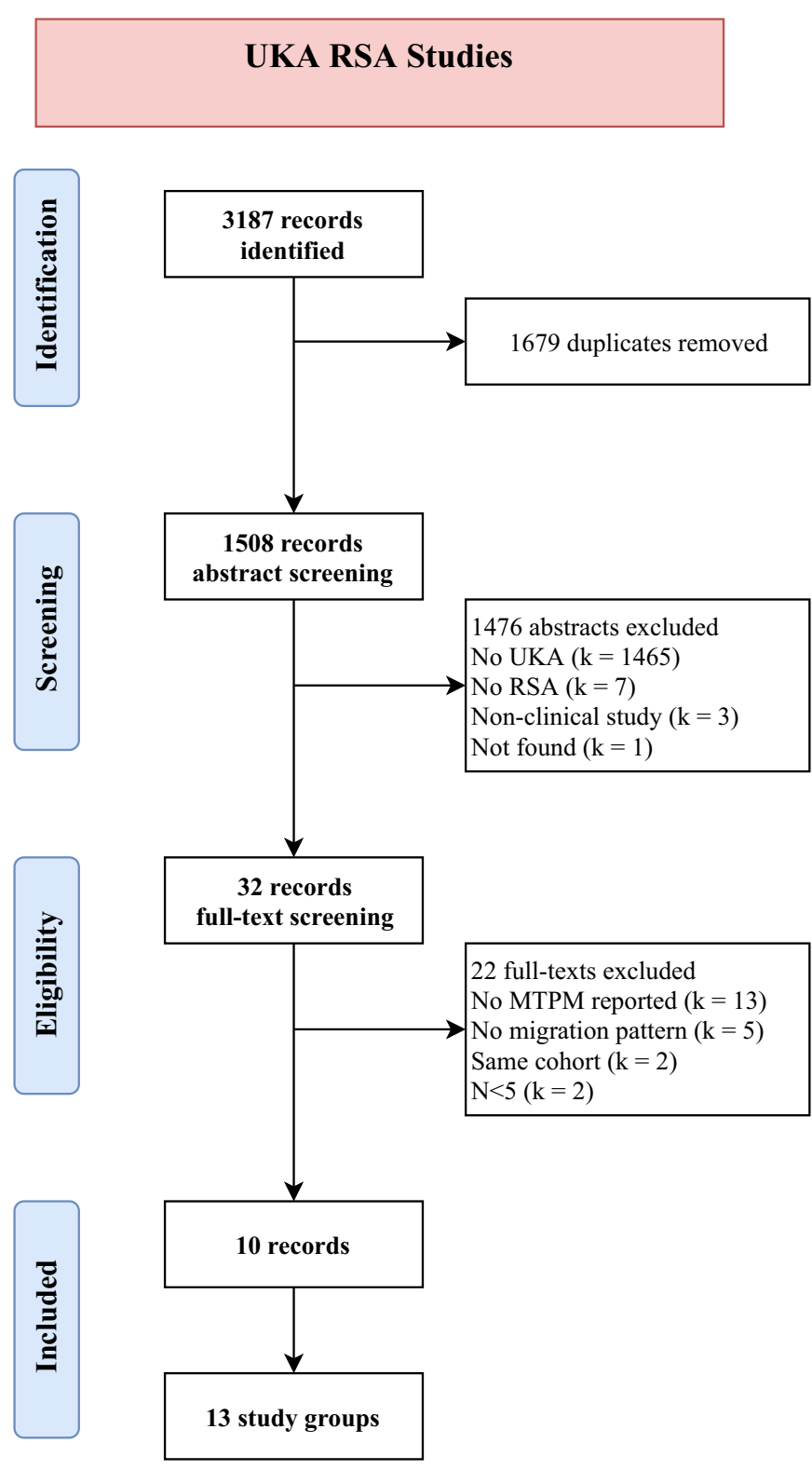

Fig. 1. PRISMA flowchart. Illustrating the selection and inclusion process of the review. UKA: unicondylar knee arthroplasty; TKA: total knee arthroplasty; RSA: radiostereometric analysis; MTPM: maximum total point motion.
Appendix B. Follow-up was predefined in all studies, and almost all studies $(\mathrm{k}=9)$ included more than 20 UKA. None of the studies were excluded based on the risk of bias.

Migration results

Ten studies comprising thirteen study groups were included (Table 1) [12-21]. The number of UKAs per study group ranged between 12 and 53 , with a median of 24 . All implants were cemented. The pooled mean migration of all UKAs at 3-4 months, 6 months, 1 year, and 2 years was $0.43 \mathrm{~mm}$ ( $95 \%$ CI 0.38 to 0.48 ), $0.54 \mathrm{~mm}$ ( $95 \%$ CI 0.40 to 0.67 ), $0.59 \mathrm{~mm}$ ( $95 \%$ CI 0.52 to 0.66 ), and $0.61 \mathrm{~mm}$ (95\% CI 0.55 to 0.68 ), respectively (Figs. 2 and 3). UKAs migrated predominately in the first 3-4 months. The migration of UKAs at 1 year and 2 years was higher than the migration of TKAs at these time points (Fig. 3) [7]. The increase in migration between 6 months and 1 year was $0.17 \mathrm{~mm} \mathrm{(95 \%} \mathrm{CI} 0.03$ to 0.32 ) based on five study groups. After 1 year, there was little migration: the pooled increase in MTPM migration between 1 year and 2 years was $0.05 \mathrm{~mm}$ ( $95 \%$ CI -0.03 to 0.12 ) based on 13 study groups. This increase was comparable to the increase of TKAs between 1 year and 2 years: $0.04 \mathrm{~mm}$ (95\% CI 0.02-0.06) [7]. Secondary migration of MBT and APT components was compared. MBT and APT UKAs showed a comparable migration pattern up to 6 months of follow-up, but APT implants had a statistically significant higher MTPM at 1 year $(\mathrm{p}=0.007)$, while this difference was less prominent at 2 years $(\mathrm{p}=0.09$ ) (Fig. 4). The influence of publication year on migration was plotted post-hoc. This figure suggests that the migration of metal-backed UKAs has decreased over time in contrast to that of all-polyethylene UKAs (Fig. 5).

\section{Discussion}

The present review found that the migration profile of UKAs and found that these were comparable with the migration profile of TKAs, with high initial migration and little migration between 1 year and 2 years. However, UKAs had a higher initial migration at 1 year and 2 years compared with TKAs. In addition, APT UKAs migrated more than MBT UKAs. Last, a trend towards a decrease of migration of MBT UKAs over the past three decades was found.

These findings suggest that the threshold used to identify implants at risk for early loosening (i.e., $>0.2$ MTPM from the first to the second postoperative year) could be used for UKAs, as has been done in several UKA RSA studies [13,15-17,21]. Besides this threshold, Pijls et al. (2012) proposed a classification of TKAs into three groups based on 1-year MTPM and long-term implant survival of TKAs: $<0.54 \mathrm{~mm} 1$-year MTPM (i.e., acceptable), 0.54-1.6 mm 1-year MTPM (i.e., at risk), and $>1.6 \mathrm{~mm}$ 1-year MTPM (i.e., unacceptable) [7]. If this classification was to be used to classify the included UKAs of the present meta-analysis, three UKAs would have been classified as acceptable, ten as at risk, and none as unacceptable. However, the 1-year MTPM was higher for UKAs compared with TKAs, which would naturally result in a higher number of UKAs being classified as at risk or unacceptable. Whether it is justified to use this classification for UKAs remains unclear, and long-term RSA UKA studies are needed to address this question. Moreover, the clinical relevance of the found difference in migration between UKAs and TKAs is unclear and should be studied further.

Our review did not include any migration studies comprising uncemented UKAs. There was one RSA study comparing uncemented and cemented UKAs, but that study did not report MTPM and was therefore not included [22]. That study found comparable migration of uncemented and cemented UKAs [22]. Kerens et al. (2017) found no difference in revision or clinical scores but found fewer radiolucent lines and a shorter operative time for uncemented UKAs [23], while two recent registry studies involving 14,814 and 8,733 knees found a lower revision rate for uncemented UKAs compared with cemented UKAs [24,25]. The popularity of uncemented UKAs is increasing: in the Dutch arthroplasty register, $3 \%$ of UKAs were uncemented in 2010 , compared with $54 \%$ in 
Table 1

Study characteristics of RSA studies.

\begin{tabular}{|c|c|c|c|c|c|c|c|}
\hline \multirow[t]{2}{*}{ Author (Year) } & \multirow[t]{2}{*}{ Unicondylar knee arthroplasty } & \multirow{2}{*}{$\begin{array}{l}\text { Patients } \\
\text { Total, } \\
\mathrm{N}\end{array}$} & \multirow{2}{*}{$\begin{array}{l}\text { Age } \\
\text { Mean, } \\
\text { years }\end{array}$} & \multirow{2}{*}{$\begin{array}{l}\text { MTPM } \\
3-4 \text { months } \\
\begin{array}{l}\text { Mean, } \\
\text { millimeters (se) }\end{array}\end{array}$} & \multirow{2}{*}{$\begin{array}{l}\text { MTPM } \\
6 \text { months } \\
\text { Mean, } \\
\text { millimeters (se) }\end{array}$} & \multirow{2}{*}{$\begin{array}{l}\text { MTPM } \\
1 \text { year } \\
\begin{array}{l}\text { Mean, } \\
\text { millimeters (se) }\end{array}\end{array}$} & \multirow{2}{*}{$\begin{array}{l}\text { MTPM } \\
2 \text { years } \\
\text { Mean, } \\
\text { millimeters (se) }\end{array}$} \\
\hline & & & & & & & \\
\hline $\begin{array}{l}\text { Linde (2019) } \\
\quad[17]\end{array}$ & $\begin{array}{l}\text { Oxford Phase III cemented medial mobile-bearing } \\
\text { metal-backed tibial component }\end{array}$ & 53 & 65 & & & $0.47(0.04)$ & $0.54(0.05)$ \\
\hline \multirow[t]{2}{*}{$\begin{array}{l}\text { Koppens (2019) } \\
\text { [16] }\end{array}$} & $\begin{array}{l}\text { Oxford Phase III cemented medial mobile-bearing } \\
\text { metal-backed tibial component }\end{array}$ & 33 & 64 & $0.42(0.06)$ & & $0.54(0.07)$ & $0.55(0.06)$ \\
\hline & $\begin{array}{l}\text { Sigma cemented medial fixed-bearing metal-backed } \\
\text { tibial component }\end{array}$ & 32 & 61 & $0.44(0.06)$ & & $0.51(0.08)$ & $0.50(0.06)$ \\
\hline $\begin{array}{l}\text { Koppens (2018) } \\
\text { [15] }\end{array}$ & $\begin{array}{l}\text { Sigma High-Performance cemented medial fixed- } \\
\text { bearing metal-backed tibial component }\end{array}$ & 45 & 64 & $0.47(0.08)$ & & $0.53(0.09)$ & $0.65(0.09)$ \\
\hline $\begin{array}{l}\text { Bruni (2015) } \\
\quad[13]\end{array}$ & $\begin{array}{l}\text { Duracon cemented medial/lateral (unclear) (fixed) } \\
\text { bearing all-polyethylene tibial component }\end{array}$ & 36 & 71 & & & $1.12(0.27)$ & $1.35(0.39)$ \\
\hline $\begin{array}{l}\text { Ensini (2013) } \\
\quad[21]\end{array}$ & $\begin{array}{l}\text { Optetrak cemented medial (fixed) bearing all- } \\
\text { polyethylene tibial component }\end{array}$ & 23 & 69 & $0.46(0.08)$ & $0.67(0.08)$ & $0.70(0.14)$ & $0.77(0.17)$ \\
\hline $\begin{array}{l}\text { Bragonzoni } \\
\quad(2005)[12]\end{array}$ & $\begin{array}{l}\text { Duracon cemented medial+lateral all-polyethylene } \\
\text { tibial component }\end{array}$ & 16 & NR & $0.47(0.09)$ & $0.55(0.08)$ & $0.61(0.16)$ & $0.67(0.18)$ \\
\hline $\begin{array}{l}\text { Soavi (2002) } \\
\quad[20]\end{array}$ & $\begin{array}{l}\text { Duracon cemented medial all-polyethylene tibial } \\
\text { component }\end{array}$ & 20 & 72 & & & $0.60(0.15)$ & $0.60(0.17)$ \\
\hline \multirow[t]{2}{*}{$\begin{array}{l}\text { Hyldahl (2001) } \\
\text { [14] }\end{array}$} & $\begin{array}{l}\text { Miller-Galante cemented medial metal-backed } \\
\text { tibial component }\end{array}$ & 18 & NR & & & $0.61(0.10)$ & $0.96(0.20)$ \\
\hline & $\begin{array}{l}\text { Miller-Galante cemented medial all-polyethylene } \\
\text { tibial component }\end{array}$ & 20 & NR & & & $0.78(0.14)$ & $0.78(0.14)$ \\
\hline $\begin{array}{l}\text { Lindstrand } \\
\text { (2000) [18] }\end{array}$ & $\begin{array}{l}\text { Duracon cemented medial+lateral all-polyethylene } \\
\text { tibial component }\end{array}$ & 49 & 72 & $0.39(0.04)$ & $0.41(0.03)$ & $0.65(0.10)$ & $0.61(0.07)$ \\
\hline \multirow[t]{2}{*}{ Ryd (1992) [19] } & $\begin{array}{l}\text { Marmor cemented medial+lateral metal-backed } \\
\text { tibial component }\end{array}$ & 24 & NR & & $0.76(0.12)$ & $0.95(0.23)$ & $1.09(0.24)$ \\
\hline & $\begin{array}{l}\text { Lund tibial component cemented medial+lateral } \\
\text { metal-backed tibial component }\end{array}$ & 12 & NR & & $0.39(0.08)$ & $0.77(0.23)$ & $0.80(0.26)$ \\
\hline
\end{tabular}

RSA: radiostereometric analysis; MTPM: maximum total point motion; se: standard error; NR: not reported.

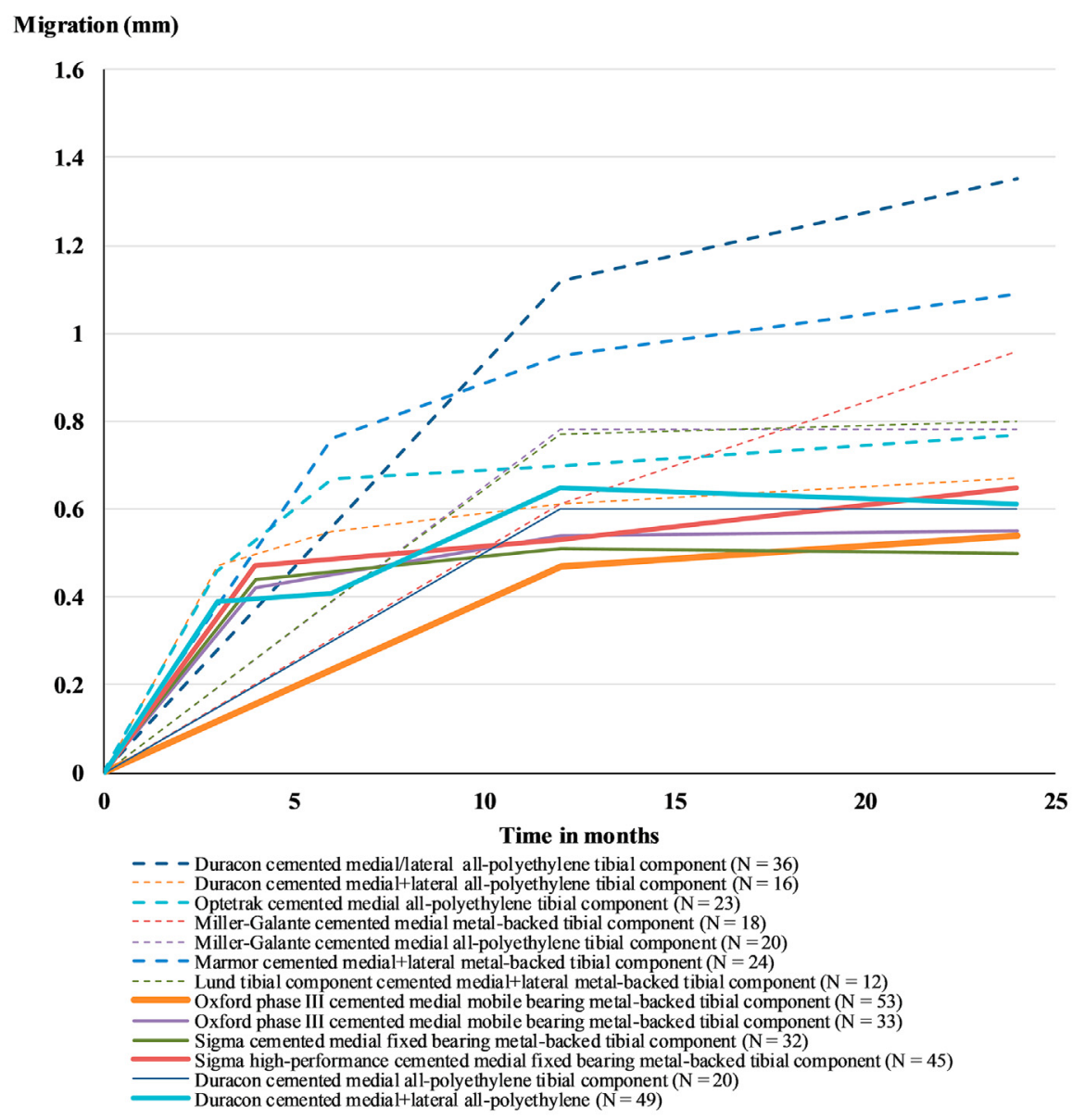

Fig. 2. Migration patterns of the included UKAs. The mean maximum total point motion (MTPM) of the included UKA by group is shown over a 2-year period. The thickness of the lines are relative to the number of patients included, with larger studies having thicker lines and smaller studies having thinner lines. Number of included patients, mean age, and mean MTPM with standard errors are reported in Table 1. UKA: unicondylar knee arthroplasty. 


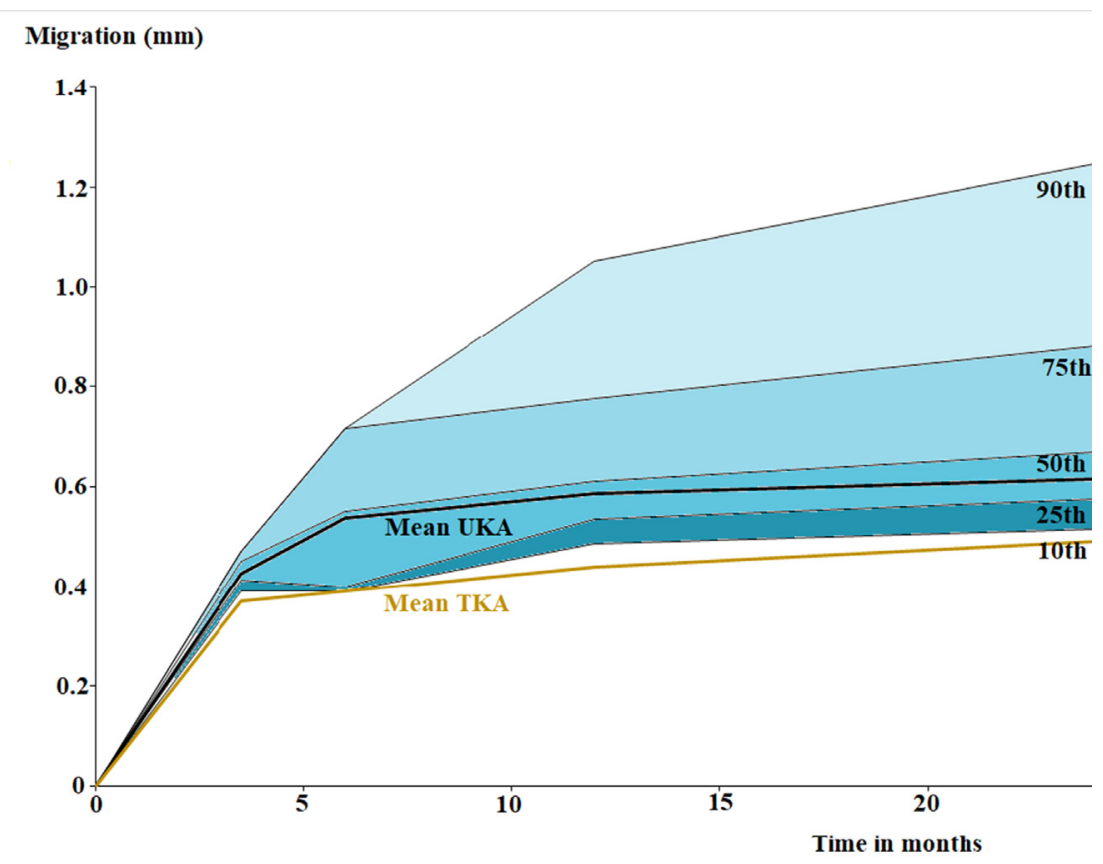

Fig. 3. Mean migration of UKAs. Mean migration is expressed as the maximum total point motion in millimetres. The bold line represents the pooled mean of all UKA, and the other lines represent the $10^{\text {th }}, 25^{\text {th }}, 50^{\text {th }}, 75^{\text {th }}$, and $90^{\text {th }}$ percentiles. The yellow line represents the mean migration of cemented TKA as previously reported [7]. UKA: unicondylar knee arthroplasty; TKA: total knee arthroplasty.

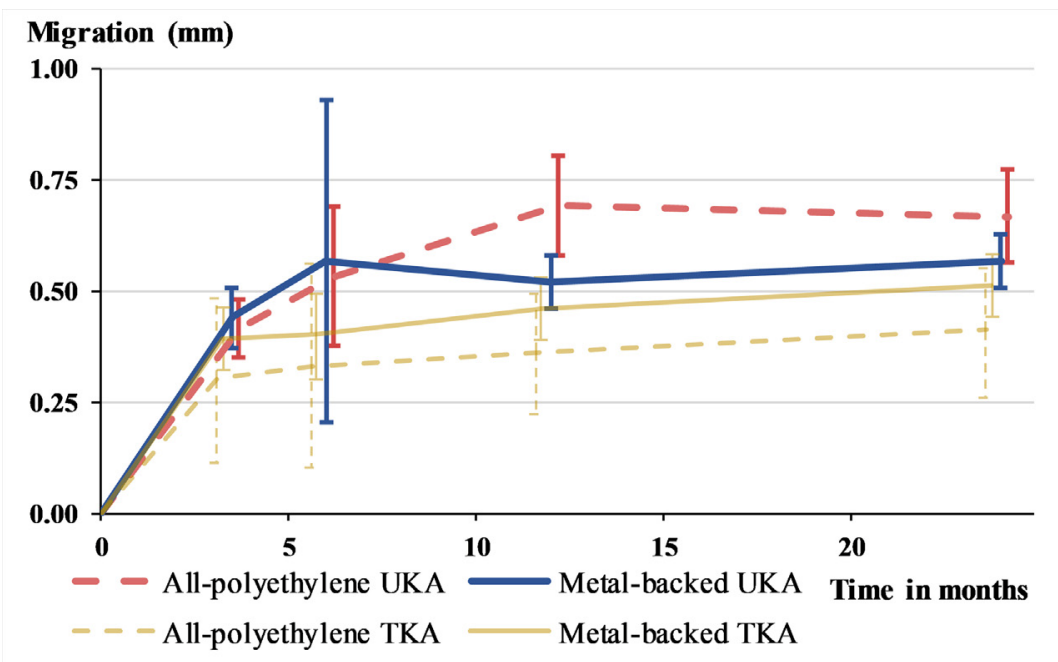

Fig. 4. Migration patterns of metal-backed and allpolyethylene UKAs. Mean migration with 95\% CIs (vertical error bars) is expressed as the maximum total point motion in millimetres ( $\mathrm{mm}$ ) of all-polyethylene and metal-backed UKAs over a 2-year period. The red, interrupted line represents the all-polyethylene UKAs, and the blue, continuous line represents the metal-backed UKAs. The yellow, interrupted line represents the mean migration of cemented all-polyethylene TKAs, and the yellow, solid line represents the mean migration of cemented metal-backed TKAs as previously reported [7]. UKA: unicondylar knee arthroplasty; TKA: total knee arthroplasty.
2019 [26]. RSA studies assessing the migration of uncemented UKAs are thus required, especially for new UKAs or UKAs without long-term follow-up either in registries or published studies.

The results of our review showed that APT UKAs migrated more than MBT UKAs at 1 year and 2 years of follow-up. This finding is in line with the results from a recent meta-analysis, which found a 2.13 higher risk of all-cause revision and a 1.66 higher risk for revision due to aseptic loosening for APT compared with MBT [27].

Several UKA designs are available, and only some of these UKA designs have been evaluated with RSA and were included in this systematic review. The 5-year all-cause revision rates of these UKAs varies between 3.3\% and $16.8 \%[2,26]$. In consideration of this variation in revision rates, a phased introduction is especially needed for UKAs to ensure patient safety. It is, therefore, highly recommended to test novel UKA designs with RSA in addition to cohort studies for mid-term and long-term survival [28-30].
Some limitations of this review should be considered. First, the number of included RSA studies was small in comparison with that in a previous review on TKAs. Ten RSA studies compared to 50 RSA studies for TKAs $[4,7]$ were included. In order to obtain a better understanding of the migration profiles of different implant designs, studies, including novel UKA designs, are needed. Moreover, some studies used medial and lateral UKAs or did not specify whether medial or lateral UKAs were included without further specifying outcomes for the medial and lateral UKAs separately. Future studies of UKAs should clearly specify the design, fixation method, insert, and anatomical compartment in order to allow for comparison between studies and to facilitate future systematic reviews. Last, the present review did not assess the influence of specific patient characteristics or surgical technique on migration, nor was the influence of publication year statistically analysed as the number of groups was limited and further subgroup analysis was deemed 


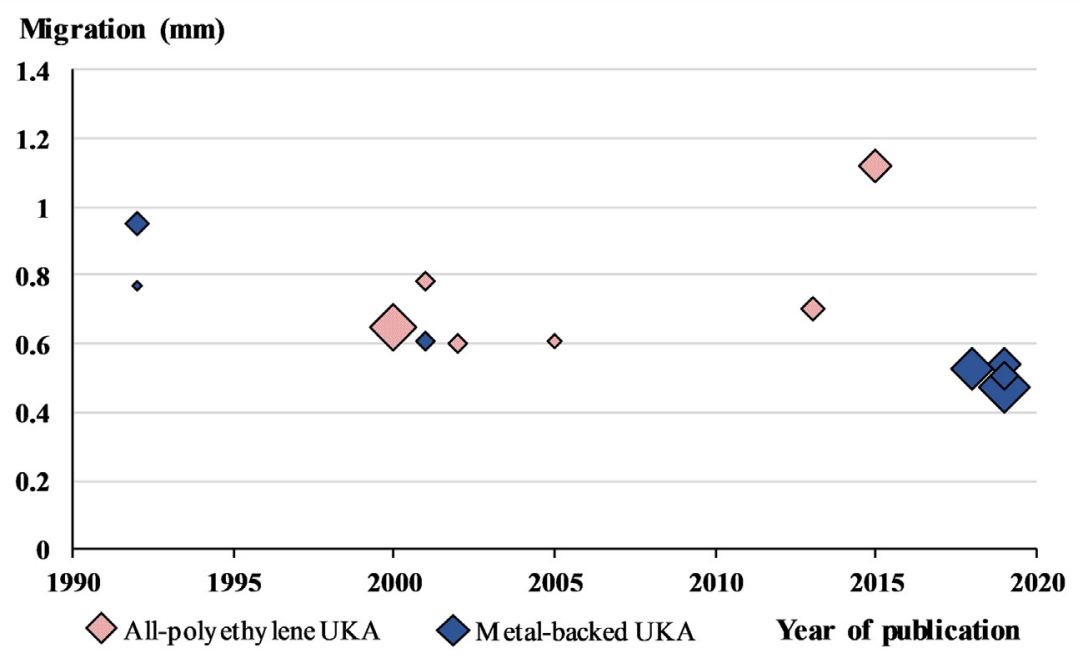

Fig. 5. UKA migration at 1 year over time. Mean migration is expressed as the maximum total point motion at 1 year in millimetres (mm) presented over time by publication year. Diamond size corresponds to the number of included patients, with larger diamonds having more patients than smaller diamonds. The red diamonds represent all-polyethylene UKAs, and the blue diamonds represent metal-backed UKAs. UKA: unicondylar knee arthroplasty.

inappropriate. Future studies should pool individual patient data to assess the influence of patient characteristics on UKA migration.

\section{Conclusion}

The migration pattern of UKAs is comparable to that of TKAs in the first two years as both show initial migration in the first few months and limited migration hereafter. However, UKAs had higher migration at one year and two years.

\section{Level of evidence}

Level II.

\section{Funding}

None.

\section{Registration}

Not registered.

\section{Author contributions}

SH: Design, formal analysis, investigation, writing-original draft preparation, writing-review and editing, visualization.

LD: Design, formal analysis, investigation, writing-original draft preparation, writing-review and editing, visualization.

RN: Conceptualization, Design, Resources, writing-review and editing, supervision, visualization.

JP: Design, Investigation, resources, writing-review and editing.

LB: Conceptualization, Design, writing-review and editing, supervision, visualization.

BP: Conceptualization, Design, formal analysis, investigation, writing-original draft preparation, writing-review and editing, supervision, visualization.

\section{Declaration of competing interest}

Dr. Blankevoort has a patent WO2017105232A1 issued. Other authors did not declare a competing interest.

\section{Appendix A. Supplementary data}

Supplementary data to this article can be found online at https://doi. org/10.1016/j.jisako.2021.12.002.

\section{References}

[1] Johal S, Nakano N, Baxter M, Hujazi I, Pandit H, Khanduja V. Unicompartmental knee arthroplasty: the past, current controversies, and future perspectives. J Knee Surg 2018;31(10):992-8. https://doi.org/10.1055/s-0038-1625961.

[2] NJR. N.J.R. 17th annual report 2020. 2020. 08-02-2021]; Available from: https://r eports.njrcentre.org.uk/Portals/0/PDFdownloads/NJR\%2017th\%20Annual\% 20Report\%202020.pdf.

[3] Wilson HA, Middleton R, Abram SGF, et al. Patient relevant outcomes of unicompartmental versus total knee replacement: systematic review and metaanalysis. BMJ 2019;364:1352. https://doi.org/10.1136/bmj.1352.

[4] Pijls BG, Valstar ER, Nouta KA, et al. Early migration of tibial components is associated with late revision: a systematic review and meta-analysis of 21,000 knee arthroplasties. Acta Orthop 2012;83(6):614-24. https://doi.org/10.3109/ 17453674.2012.747052.

[5] Ryd L, Albrektsson BE, Carlsson L, et al. Roentgen stereophotogrammetric analysis as a predictor of mechanical loosening of knee prostheses. J Bone Joint Surg Br 1995;77(3):377-83. pmid: 7744919.

[6] Page MJ, McKenzie JE, Bossuyt PM, et al. The PRISMA 2020 statement: an updated guideline for reporting systematic reviews. PLoS Med 2021;18(3):e1003583. https://doi.org/10.1371/journal.pmed.1003583.

[7] Pijls BG, Plevier JWM, Nelissen R. RSA migration of total knee replacements. Acta Orthop 2018;89(3):320-8. https://doi.org/10.1080/17453674.2018.1443635.

[8] van Hamersveld KT, Marang-van de Mheen PJ, Koster LA, Nelissen R, ToksvigLarsen S, Kaptein BL. Marker-based versus model-based radiostereometric analysis of total knee arthroplasty migration: a reanalysis with comparable mean outcomes despite distinct types of measurement error. Acta Orthop 2019;90(4): 366-72. https://doi.org/10.1080/17453674.2019.1605692.

[9] Pijls BG, Dekkers OM, Middeldorp S, et al. AQUILA: assessment of quality in lower limb arthroplasty. An expert Delphi consensus for total knee and total hip arthroplasty. BMC Muscoskel Disord 2011;12:173. https://doi.org/10.1186/14712474-12-173.

[10] Viechtbauer W. Conducting meta-analyses in R with metafor package. Journal of Statostical Software 2010;36(3):1-48. https://doi.org/10.18637/jss.v036.i03.

[11] Weir CJ, Butcher I, Assi V, et al. Dealing with missing standard deviation and mean values in meta-analysis of continuous outcomes: a systematic review. BMC Med Res Methodol 2018;18(1):25. https://doi.org/10.1186/s12874-018-0483-0.

[12] Bragonzoni L, Russo A, Loreti I, Montagna L, Visani A, Marcacci M. The stressinducible displacement detected through RSA in non-migrating UKR. Knee 2005; 12(4):301-6. https://doi.org/10.1016/j.knee.2004.09.006.

[13] Bruni D, Bragonzoni L, Gagliardi M, et al. Roentgen stereophotogrammetric analysis: an effective tool to predict implant survival after an all-poly unicompartmental knee arthroplasty-a 10 year follow-up study. Knee Surg Sports Traumatol Arthrosc 2015;23(11):3273-80. https://doi.org/10.1007/s00167-0143106-2.

[14] Hyldahl HC, Regnér L, Carlsson L, Kärrholm J, Weidenhielm L. Does metal backing improve fixation of tibial component in unicondylar knee arthroplasty? A 
randomized radiostereometric analysis. J Arthroplasty 2001;16(2):174-9. https:// doi.org/10.1054/arth.2001.20257.

[15] Koppens D, Stilling M, Munk S, et al. Low implant migration of the SIGMA(®) medial unicompartmental knee arthroplasty. Knee Surg Sports Traumatol Arthrosc 2018;26(6):1776-85. https://doi.org/10.1007/s00167-017-4782-5.

[16] Koppens D, Rytter S, Munk S, et al. Equal tibial component fixation of a mobilebearing and fixed-bearing medial unicompartmental knee arthroplasty: a randomized controlled RSA study with 2-year follow-up. Acta Orthop 2019;90(6): 575-81. https://doi.org/10.1080/17453674.2019.1639965.

[17] Linde KN, Madsen F, Puhakka KB, et al. Preoperative systemic bone quality does not affect tibial component migration in knee arthroplasty: a 2-year radiostereometric analysis of a hundred consecutive patients. J Arthroplasty 2019;34(10):2351-9. https://doi.org/10.1016/j.arth.2019.05.019.

[18] Lindstrand A, Stenström A, Ryd L, Toksvig-Larsen S. The introduction period of unicompartmental knee arthroplasty is critical: a clinical, clinical multicentered, and radiostereometric study of 251 Duracon unicompartmental knee arthroplasties. J Arthroplasty 2000;15(5):608-16. https://doi.org/10.1054/arth.2000.6619.

[19] Ryd L, Lindstrand A, Stenström A, Selvik G. The influence of metal backing in unicompartmental tibial component fixation. An in vivo roentgen stereophotogrammetric analysis of micromotion. Arch Orthop Trauma Surg 1992; 111(3):148-54. https://doi.org/10.1007/BF00388089.

[20] Soavi R, Loreti I, Bragonzoni L, La Palombara PF, Visani A, Marcacci M. A roentgen stereophotogrammetric analysis of unicompartmental knee arthroplasty. J Arthroplasty 2002;17(5):556-61. https://doi.org/10.1054/arth.2002.32139.

[21] Ensini A, Barbadoro P, Leardini A, Catani F, Giannini S. Early migration of the cemented tibial component of unicompartmental knee arthroplasty: a radiostereometry study. Knee Surg Sports Traumatol Arthrosc 2013;21(11):2474-9. https://doi.org/10.1007/s00167-012-2068-5.

[22] Kendrick BJ, Kaptein BL, Valstar ER, et al. Cemented versus cementless Oxford unicompartmental knee arthroplasty using radiostereometric analysis: a randomised controlled trial. Bone Joint J 2015;97-b(2):185-91. https://doi.org/ 10.1302/0301-620X.97B2.34331.

[23] Kerens B, Schotanus MGM, Boonen B, et al. Cementless versus cemented Oxford unicompartmental knee arthroplasty: early results of a non-designer user group. Knee Surg Sports Traumatol Arthrosc 2017;25(3):703-9. https://doi.org/10.1007/ s00167-016-4149-3.

[24] Mohammad HR, Matharu GS, Judge A, Murray DW. Comparison of the 10-year outcomes of cemented and cementless unicompartmental knee replacements: data from the National Joint Registry for England, Wales, Northern Ireland and the Isle of Man. Acta Orthop 2020;91(1):76-81. https://doi.org/10.1080/ 17453674.2019.1680924.

[25] Gupta V, Kejriwal R, Frampton C. Revision following cemented and uncemented oxford-III primary medial unicompartmental knee replacements: a 19-year analysis from the New Zealand joint registry. J Bone Joint Surg Am 2020;102(20):1777-83. https://doi.org/10.2106/JBJS.19.01443.

[26] Dutch Arthroplasty Register (LROI). Online LROI annual report 2020. 2020 11-032021]; Available from: https://www.lroi-report.nl/.

[27] Costa GG, Lo Presti M, Grassi A, et al. Metal-backed tibial components do not reduce risk of early aseptic loosening in unicompartmental knee arthroplasty: a systematic review and meta-analysis. J Knee Surg 2020;33(2):180-9. https://doi.org/ 10.1055/s-0038-1677506.

[28] Sedrakyan A, Campbell B, Merino JG, Kuntz R, Hirst A, McCulloch P. IDEAL-D: a rational framework for evaluating and regulating the use of medical devices. BMJ 2016;353:i2372. https://doi.org/10.1136/bmj.i2372.

[29] Nelissen RG, Pijls BG, Karrholm J, Malchau H, Nieuwenhuijse MJ, Valstar ER. RSA and registries: the quest for phased introduction of new implants. J Bone Joint Surg Am 2011;93(Suppl 3):62-5. https://doi.org/10.2106/JBJS.K.00907.

[30] Malchau H. On the importance of stepwise introduction of new hip implant technology. Thesis. Göteborg University; 1995. 